\title{
PURCHASING POWER PARITY TESTS IN COINTEGRATED PANELS
}

\author{
Peter Pedroni*
}

\begin{abstract}
This paper employs recently developed techniques for testing hypotheses in cointegrated panels to test the strong version of purchasing power parity for a panel of post Bretton Woods data. We compare results using fully modified and dynamic OLS approaches, and strongly reject the hypothesis. We also introduce a new between-dimension dynamic OLS estimator and find that the between-dimension FMOLS and DOLS estimates of the long-run deviation from purchasing power parity are larger than the correspondin $g$ within-dimension estimates. Finally, we attempt to reconcile these rejections with the mixed findings that have been reported in panel unit root studies.
\end{abstract}

\section{Introduction}

A LTHOUGH long-run purchasing power parity (PPP) has been relatively easy to evidence for exchange rates that span long periods of time, it has been considerably more difficult to find such evidence for the relatively short spans of data corresponding to the recent floating exchange rate period that followed the collapse of the Bretton Woods system in 1973. Consequently, studies such as Frankel and Rose (1995), O'Connell (1998), Oh (1996), Papell (1997), and $\mathrm{Wu}$ (1996) examine whether PPP appears to hold under the recent float on the basis of what have been interpreted as more powerful panel unit root tests of the real exchange rate. The results have been somewhat mixed, however, and tend to be sensitive to the inclusion of different subsets of the panel.

In assessing this evidence, it is important to realize that panel unit root studies impose a homogeneous unit value for the implied cointegrating vector between nominal exchange rates and aggregate price ratios. But many authors have argued that, although there may be a tendency for these variables to move together in equilibrium over long periods, the relationship need not necessarily be one-for-one under more general interpretations known as "weak" PPP. Examples of mechanisms that can induce such circumstances include differences in price indices between countries, measurement errors, transportation costs, and differential productivity shocks. In the context of panels, it is quite natural to imagine that, if these factors play a role in the data, they

Received for publication September 23, 1997. Revision accepted for publication September 27, 2000.

* Indiana University.

Some of the findings in this paper first appeared in a working paper entitled "Panel Fully Modified OLS for Heterogeneou s Cointegrated Panels and the Case of Purchasing Power Parity," Indiana University (June 1996). The econometric theory and Monte Carlo portion of the working paper are available in Advances in Econometrics, vol. 15. I thank especially Bob Cumby, Pravin Trivedi, three anonymous referees, and James Stock as editor for helpful comments and suggestion s on various earlier versions. This study has also benefitted from presentation at the North American Econometric Society Summer Meetings (June 1996), the Midwest International Economics Meetings (April 1996), and workshop seminars at Rice University-Universit y of Houston, Southern Methodist University, The Federal Reserve Bank of Kansas City, U.C. Santa Cruz, and Washington University. A computer program that implements the between-dimension DOLS procedure introduced in this paper is available upon request from the author. are also just as likely to be of varying significance across differing countries, so that one should therefore be prepared for the possibility of heterogeneous cointegrating relationships. Consequently, by using the panel cointegration methods developed in Pedroni (1995) that allow one to test the null of no cointegration without imposing homogeneity of the cointegrating vector, studies by Canzoneri, Cumby, and Diba (1999), Chinn (1997), Obstfeld and Taylor (1996), Pedroni (1995), and Taylor (1996) all find support for this weaker version of PPP with heterogeneous slope coefficients.

Such results in favor of weak PPP do not contradict the stronger version per se, but they do suggest an interpretation for the mixed findings in tests of strong PPP based on panel unit root tests of the real exchange rate. Specifically, in the panel setting, if the maintained hypothesis that the cointegrating vector is homogeneous and equal to one for all countries is violated even for a small subset, because this mixes a few integrated series in with the majority of stationary ones, this is likely to lead to an inability to reject the null of a unit root for the panel. Conversely, because of the way the null hypothesis is constructed for the panel, a rejection of the unit root null simply implies that the data from at least some countries are consistent with PPP. It does not imply that the data from all countries of the sample are consistent with PPP, which would appear to be the more natural way to pose the question.

By contrast, the approach in this paper allows us to directly test the condition on the cointegrating vector that is required for strong PPP to hold. Furthermore, it allows us to pose the null hypothesis in a more natural form, so that we test whether or not strong PPP holds consistently for all countries of the panel. By comparing results for individual countries and the panel as a whole, we firmly reject the hypothesis and confirm that the rejection is not due to only a few countries, but rather that failure of strong PPP is pervasive in the post Bretton Woods period.

\section{Estimators and Test Statistics for Cointegration Vectors in Heterogeneous Panels}

When applying cointegration tests to long-run hypotheses such as PPP for aggregate panel data, a primary concern is to construct the estimators in a way that does not constrain the transitional dynamics to be similar among different countries of the panel. Instead, we would like to pool only the information concerning the long-run hypothesis of interest, and allow the short-run dynamics to be potentially heterogeneous. This was a central theme for the panel fully modified OLS tests that were developed in Pedroni (1996). In particular, Pedroni derived the asymptotic distributions and studied the small-sample properties of three versions of such estimators. Two of these-the residual-FM, and the 
adjusted-FM, pooled the data along the within-dimension, and one of these, the group-FM, pooled the data along the between-dimension. In a later version of the study, Pedroni (2000) emphasized the group-FM estimator, and found that, in contrast to the residual-FM and adjusted-FM test statistics, the group-FM exhibited relatively minor size distortions in small samples.

Kao and Chiang (1997) proposed a parametric DOLSbased panel estimator pooled along the within-dimension, and showed that it had the same asymptotic distribution as the panel FMOLS estimator studied by Pedroni (1996). Kao and Chiang also studied the small-sample properties of the panel DOLS $t$-statistic and compared it to a version of the adjusted-FM $t$-statistic that employed a first-stage OLS estimate of the cointegrating vector for the required adjustment term. In a series of Monte Carlo simulations, they found that the panel DOLS $t$-statistic had smaller size distortions than this form of the adjusted-FM $t$-statistic, although the distortion in both cases was still relatively large. More recently, Mark and Sul (1999) proposed a variation to the panel DOLS estimator and showed that it somewhat improved the small-sample performance, although even these small-sample size distortions are still fairly large. In comparing the two, Kao and Chiang's panel DOLS estimator can be viewed as a weighted estimator, and Mark and Sul's panel DOLS estimator can be viewed as an unweighted estimator. The asymptotics in all of these studies were based on sequential limits. By contrast, Phillips and Moon (1999) developed an asymptotic theory for nonstationary panels based on joint limits, and were able to show that a version of the within-dimension panel FMOLS estimator converges jointly in distribution.

In this study, we employ both the within-dimension and between-dimension panel FMOLS tests from Pedroni (1996, 2000). We also employ the weighted panel DOLS estimator from Kao and Chiang (1997) and the unweighted panel DOLS estimator from Mark and Sul (1999). However, both of these DOLS estimators are within-dimension estimators. Thus, for comparison with the between-dimension "group-mean" panel FMOLS estimator, we also introduce here an analogous between-dimension, group-mean panel DOLS estimator.

An important advantage of the between-dimension estimators is that the form in which the data is pooled allows for greater flexibility in the presence of heterogeneity of the cointegrating vectors. Specifically, whereas test statistics constructed from the within-dimension estimators are designed to test the null hypothesis $H_{o}: \beta_{i}=\beta_{o}$ for all $i$ against the alternative hypothesis $H_{A}: \beta_{i}=\beta_{A} \neq \beta_{o}$ where the value $\beta_{A}$ is the same for all $i$, test statistics constructed from the between-dimension estimators are designed to test the null hypothesis $H_{o}: \beta_{i}=\beta_{o}$ for all $i$ against the alternative hypothesis $H_{A}: \beta_{i} \neq \beta_{o}$, so that the values for $\beta_{i}$ are not constrained to be the same under the alternative hypothesis. Clearly, this is an important advantage for applications such as the present one, because there is no reason to believe that, if the cointegrating slopes are not equal to one, that they necessarily take on some other arbitrary common value. Another advantage of the betweendimension estimators is that the point estimates have a more useful interpretation in the event that the true cointegrating vectors are heterogeneous. Specifically, point estimates for the between-dimension estimator can be interpreted as the mean value for the cointegrating vectors. This is not true for the within-dimension estimators.

Finally, the test statistics constructed from the groupmean estimators appear to have another advantage even under the null hypothesis when the cointegrating vector is homogeneous. Specifically, Pedroni (2000) shows that they appear to suffer from much lower small-sample size distortion than the within-dimension estimators. A similar analysis of the between-dimension, group-mean panel DOLS that is introduced here reveals that it also exhibits much less size distortion relative to the within-dimension panel DOLS estimators. In the interest of space we do not report these, and leave a more thorough study of the small-sample properties for future research.

Because we emphasize here the FM and DOLS betweendimension estimators, and because the between-dimension DOLS estimator is new to this paper, we restrict ourselves here to describing these two estimators and refer the reader to the original works for the within-dimension estimators. Thus, consider the regression

$$
s_{i t}=\alpha_{i}+\beta_{i} p_{i t}+\mu_{i t},
$$

where $s_{i t}$ is the log bilateral U.S. nominal exchange rate, $p_{i t}$ is the log aggregate price ratio in terms of the CPI between the two countries, and

$s_{i t}$ and $p_{i t}$ are cointegrated with slopes $\beta_{i}$, which may or may not be homogeneous across $i$.

In this case, for strong PPP to hold, we require under the null hypothesis that $H_{o}: \beta_{i}=1$ for all $i$. Let $\xi_{i t}=\left(\hat{\mu}_{i t}\right.$, $\left.\Delta p_{i t}\right)^{\prime}$ be a stationary vector consisting of the estimated residuals from the cointegrating regression and the differences in prices, and let $\Omega_{i} \equiv \lim _{T \rightarrow \infty} E\left[T^{-1}\left(\sum_{t=1}^{T} \xi_{i t}\right)\right.$ $\left.\left(\sum_{t=1}^{T} \xi_{i t}^{\prime}\right)\right]$ be the long-run covariance for this vector process. This long-run covariance matrix is typically estimated using any one of a number of HAC estimators, such as the Newey-West estimator. It can be decomposed as $\Omega_{i}=\Omega_{i}^{o}$ $+\Gamma_{i}+\Gamma_{i}^{\prime}$, where $\Omega_{i}^{o}$ is the contemporaneous covariance and $\Gamma_{i}$ is a weighted sum of autocovariances.

Using this notation, we see that, based on Pedroni (1996, 2000), the expression for the between-dimension, groupmean panel FMOLS estimator is given as 


$$
\begin{aligned}
\hat{\beta}_{G F M}^{*}= & N^{-1} \sum_{i=1}^{N}\left(\sum_{t=1}^{T}\left(p_{i t}-\bar{p}_{i}\right)^{2}\right)^{-1} \\
& \times\left(\sum_{t=1}^{T}\left(p_{i t}-\bar{p}_{i}\right) s_{i t}^{*}-T \hat{\gamma}_{i}\right)
\end{aligned}
$$

where

$$
\begin{aligned}
& s_{i t}^{*}=\left(s_{i t}-\bar{s}_{i}\right)-\frac{\hat{\Omega}_{21 i}}{\hat{\Omega}_{22 i}} \Delta p_{i t}, \\
& \hat{\gamma}_{i} \equiv \hat{\Gamma}_{21 i}+\hat{\Omega}_{21 i}^{o}-\frac{\hat{\Omega}_{21 i}}{\hat{\Omega}_{22 i}}\left(\hat{\Gamma}_{22 i}+\hat{\Omega}_{22 i}^{o}\right) .
\end{aligned}
$$

Because the expression following the summation over the $i$ is identical to the conventional time series FMOLS estimator, we see that the between-dimension estimator can be constructed simply as $\hat{\beta}_{G F M}^{*}=N^{-1} \sum_{i=1}^{N} \hat{\beta}_{F M, i}^{*}$, where $\hat{\beta}_{F M, i}^{*}$ is the conventional FMOLS estimator, applied to the $i$ th member of the panel. Likewise, the associated $t$-statistic for the between-dimension estimator can be constructed as

$$
t_{\hat{\beta}_{G F M}^{*}}=N^{-1 / 2} \sum_{i=1}^{N} t_{\hat{\beta}_{F M, i}^{*}}
$$

where

$$
t_{\hat{\beta} F M, i}^{*}=\left(\hat{\beta}_{F M, i}^{*}-\beta_{o}\right)\left(\hat{\Omega}_{11 i}^{-1} \sum_{t=1}^{T}\left(p_{i t}-\bar{p}_{i}\right)^{2}\right)^{1 / 2} .
$$

In similar spirit, a between-dimension, group-mean panel DOLS estimator can be constructed as follows. First, we begin by augmenting the cointegrating regression with lead and lagged differences of the regressor to control for the endogenou s feedback effect. This plays a role that is similar to the nonparametric correction term for $s_{i t}^{*}$ in terms of $\Delta p_{i t}$ in the FMOLS procedure. Consequently, the DOLS regression becomes

$$
s_{i t}=\alpha_{i}+\beta_{i} p_{i t}+\sum_{k=-K_{i}}^{K_{i}} \gamma_{i k} \Delta p_{i t-k}+\mu_{i t}^{*}
$$

From this regression, we construct the group-mean panel DOLS estimator as

$$
\hat{\beta}_{G D}^{*}=\left[N^{-1} \sum_{i=1}^{N}\left(\sum_{t=1}^{T} z_{i t} z_{i t}^{\prime}\right)^{-1}\left(\sum_{t=1}^{T} z_{i t} \tilde{s}_{i t}\right)\right]
$$

where

$$
\begin{aligned}
& z_{i t} \text { is the } 2(K+1) \times 1 \text { vector of regressors } z_{i t}=\left(p_{i t}-\right. \\
& \left.\bar{p}_{i}, \Delta p_{i t-K}, \ldots, \Delta p_{i t+K}\right), \\
& \tilde{s}_{i t}=s_{i t}-\bar{s}_{i} \text {, and } \\
& \text { the subscript } 1 \text { outside the brackets indicates that we are } \\
& \text { taking only the first element of the vector to obtain the } \\
& \text { pooled slope coefficient. }
\end{aligned}
$$

Again, because the expression following the summation over the $i$ is identical to the conventional time series DOLS estimator, we see that the between-dimension estimator can be constructed simply as $\hat{\beta}_{G D}^{*}=N^{-1} \sum_{i=1}^{N} \hat{\beta}_{D, i}^{*}$, where $\hat{\beta}_{D, i}^{*}$ is the conventional DOLS estimator, applied to the $i$ th member of the panel. Similarly, if we let $\sigma_{i}^{2}=\lim _{T \rightarrow \infty}$ $E\left[T^{-1}\left(\sum_{t=1}^{T} \hat{\mu}_{i t}^{*}\right)^{2}\right]$ be the long-run variance of the residuals from the DOLS regression (which again can be estimated using standard HAC methods), then the associated $t$-statistic for the between-dimension estimator can be constructed as

$$
t_{\hat{\beta}_{G D}^{*}}=N^{-1 / 2} \sum_{i=1}^{N} t_{\hat{\beta}_{D, i}^{*}}
$$

where

$$
t_{\hat{\beta} \ddot{\mathrm{D}, i}}=\left(\hat{\beta}_{D, i}^{*}-\beta_{o}\right)\left(\hat{\sigma}_{i}^{-2} \sum_{t=1}^{T}\left(p_{i t}-\bar{p}_{i}\right)^{2}\right)^{1 / 2} .
$$

\section{Results}

Results are displayed in table 1 . The panel consists of 246 monthly observations of end-of-period nominal bilateral U.S. dollar exchange rates and aggregate CPI ratios for twenty countries from the IFS for which the data exists uninterrupted from June 1973 through November 1993. It is the same data set that was employed in the empirical illustration that was provided in Pedroni (1995), which used the null of no cointegration to test the weak form of PPP. Consequently, the data has already been tested to confirm that, although the unit root null could not be rejected for the panels of individual variables, the null of no cointegration is firmly rejected. Individual FMOLS and DOLS estimates and $t$-statistics for $H_{o}: \beta_{i}=1$ are reported in the first twenty entries. At the bottom of the table, results are reported for the panel estimators with and without common time dummies.

The results from both the individual tests and the panel tests overwhelmingly reject the null hypothesis of strong PPP. Among the individual country tests, data from fourteen of the twenty countries produce rejections at the $10 \%$ level for either the FMOLS or DOLS tests, and eight produce rejections at the $1 \%$ level for either test. In most cases, the results of the FMOLS and DOLS are in agreement. For the panel tests, all six reported tests reject at the $10 \%$ level or 
Table 1.-Purchasing Power Parity Tests

\begin{tabular}{lcccc}
\hline \hline Country & FMOLS & $t$-stat & DOLS & $t$-stat \\
\hline UK & 0.68 & $-2.59^{* *}$ & 0.67 & $-1.91^{*}$ \\
Belgium & 0.31 & -1.47 & 0.23 & $-1.96^{*}$ \\
Denmark & 1.63 & $1.83^{*}$ & 1.90 & $2.85^{* *}$ \\
France & 2.00 & $4.79^{* *}$ & 2.21 & $8.09^{* *}$ \\
Germany & 0.80 & -1.37 & 0.91 & -0.60 \\
Italy & 0.97 & -0.44 & 1.08 & 1.12 \\
Holland & 0.69 & $-1.83^{*}$ & 0.66 & $-2.06^{*}$ \\
Sweden & 1.22 & 1.13 & 1.16 & 0.82 \\
Switz. & 1.27 & $1.87^{*}$ & 1.36 & $2.25^{*}$ \\
Canada & 1.44 & $2.00^{*}$ & 1.43 & 1.88 \\
Japan & 1.79 & $4.94^{*}$ & 1.75 & $5.03^{* *}$ \\
Greece & 1.02 & 0.52 & 0.99 & -0.37 \\
Portugal & 1.05 & 1.30 & 1.09 & $2.46^{*}$ \\
Spain & 0.93 & -0.80 & 1.02 & 0.18 \\
Turkey & 1.10 & $6.28^{* *}$ & 1.11 & $5.84^{* *}$ \\
N. Zealand & 1.11 & $2.19^{*}$ & 1.02 & 0.61 \\
Chile & 1.21 & $9.97^{* *}$ & 1.37 & $10.95^{* *}$ \\
Mexico & 1.03 & $2.18^{*}$ & 1.03 & $3.60^{* *}$ \\
India & 2.12 & $8.12^{* *}$ & 2.06 & $7.80^{* *}$ \\
S. Korea & 0.93 & -1.03 & 0.88 & -1.46 \\
\end{tabular}

Panel Results

Without Time Dummies

$\begin{array}{lllll}\text { Within-w } & 1.05 & 7.06 * * & 1.06 & 7.80 * * \\ \text { Within-u } & 1.09 & 2.77 * * & 1.08 & 2.20^{*} \\ \text { Between } & 1.17 & 7.87 * * & 1.20 & 9.54 * *\end{array}$

With Time Dummies

\begin{tabular}{llrrr} 
& & & \\
Within-w & 1.10 & $13.77 * *$ & 1.07 & $10.59 * *$ \\
Within-u & 1.09 & $3.60 * *$ & 1.08 & $2.79 * *$ \\
Between & 1.12 & $13.02 * *$ & 1.14 & $12.76^{* *}$ \\
\hline
\end{tabular}

$t$-stats are for $H_{o}: \beta_{i}=1 . *, * *$ indicate $10 \%, 1 \%$ rejection levels.

"Within-w" reports Pedroni (1996) weighted within-dimension adjusted-FM and analogous Kao and Chiang (1997) weighted within-dimension DOLS

"Within-u" reports Mark and Sul (1999) unweighted within-dimension DOLS and an analogous unweighted FMOLS.

"Between" reports Pedroni (1996) group-mean panel FMOLS and the group-mean panel DOLS introduced in this paper.

better, and among these all but the within-dimension panel DOLS without time dummies reject at the $1 \%$ level or better. Indeed, the unweighted within-dimension $t$-statistics are consistently lower than the others. More importantly, it is interesting to note that between-dimension estimators consistently produce larger estimates than do the withindimension estimators. Because the between-dimension estimators produce consistent estimates of the average slope under the alternative hypothesis that the slopes are different from one and vary across countries whereas the withindimension estimators do not, we take these higher values to be a more accurate representation of the average long-run relationship between nominal exchange rates and aggregate price ratios. Overall, it is interesting to note that the differences tend to be greater in comparing the within- versus between-dimensions than comparing the FMOLS versus DOLS versions.

The techniques employed in this study are based on an analysis of the cointegrating relationship between $s_{i t}$ and $p_{i t}$ for each country. By contrast, they do not address cointegrating relationships that may be present for either variable between countries. In this regard, an important concern is to make certain that the transformations that are applied in the averaging across the $N$ dimension do not inadvertently destroy the cointegrating relationships that are present among the variables for each country individually, because this could invalidate the FMOLS and DOLS regressions. An obvious example can occur when all of the exchange rates of a sample are targeted relative to each other so that the $s_{i t}$ are each cointegrated to one another. In this case when time dummies are estimated, which amounts to subtracting out the average exchange rate value each period, it can be shown that these demeaned series become stationary and the cointegrating relationship is destroyed. Although some of the currencies in this study are targeted relative to each other for portions of the sample, because others float freely, subtracting out the overall sample mean does not render any of the series stationary in our case, and we confirm this empirically with unit root tests for the demeaned series. ${ }^{1}$

Another related issue is short-run, cross-sectional dependency. Common time dummies are intended to capture certain forms of cross-sectional dependency. For example, if aggregate price ratios tend to be driven by a common external disturbance (say monetary shocks in the U.S.), the price ratios will tend to be correlated across countries. Common time dummies will account for this form of dependency. On the other hand, there are likely to be other forms of dependency that time dummies cannot control. In particular, if there are dynamic feedback effects that exist between the variables of different countries, these cannot be controlled for simply by using time dummies. Although a general solution to the problem is beyond the scope of this paper, ${ }^{2}$ it is worthwhile to note that, based on the strong evidence from the individual country test results, a relatively simple method of inference can be used to infer that the results in this study are not altered by the likely presence of such cross-sectional dependencies. To see this, we note that, according to the Bonferroni inequality constraint, the marginal significance level, $P$, for a rejection of the null hypothesis applied to the panel of $N$ members is such that $P \leq \sum_{i=1}^{N} p_{i}$, where $p_{i}$ is the marginal significance level of the tests applied to the individual members of the panel. Dufour and Torres (1996) recommend using the criteria that $p_{i}=\frac{P}{N}$ to set the rejection level, in which case we require $p_{i} \leq 0.005$ for any one member of our sample to reject the null as applied to the panel as a whole at the $10 \%$ level. As Maddala and Wu (1999) point out, this type of testalthough conservative-does not rely on the independence of the individual tests, and is thus invariant to the presence

\footnotetext{
${ }^{1}$ Another issue is the possibility that the individual series do not contain exact unit roots, but rather are stationary with near unit roots. Because this may occur in some macro series, it will be interesting to know how robust these methods are to deviations from exact unit roots.

${ }_{2}^{2}$ Pedroni (1997) proposes a GLS type approach that uses an asymptotic covariance weighting matrix for semiparametric tests for unit roots and the null of no cointegration. In principle, a similar approach could be applied to the FMOLS estimator for sufficiently long panels.
} 
of any form of cross sectional dependency. For the normal distribution, absolute values in excess of 4.0 are sufficient for $p_{i} \leq 0.005$. In our sample, both the individual FMOLS and DOLS tests produce five such cases, leading us to conclude that no degree of cross-sectional dependency would be sufficient to overturn the rejection of strong PPP.

The overwhelming rejection of the strong PPP hypothesis that we observe in this study is not difficult to reconcile with the apparently contradictory results that are often found in panel unit root studies of strong PPP. Raw panel unit root tests are essentially a test for the null hypothesis that PPP does not hold in all countries. When this null is rejected, it merely implies that, at least for some countries, the data is more consistent with PPP than with the failure of PPP. By contrast, the tests exploited in this study reverse the null hypothesis. In this study, when we reject the null hypothesis, we are effectively rejecting the hypothesis that PPP holds in the countries of our sample in favor of the likelihood that it does not for at least some countries. Our analysis of the individual results furthermore indicates that this failure of strong PPP is not driven by the data from only a few countries. Rather, the failure of strong PPP appears to be pervasive in the post Bretton Woods period.

\section{REFERENCES}

Canzoneri, Matthew, Robert Cumby, and Behzad Diba, "Relative Labor Productivity and the Real Exchange Rate in the Long Run: Evidence for a Panel of OECD Countries," Journal of International Economics 47:2 (1999), 245-266.

Chinn, Menzie, "Sectoral Productivity, Government Spending and Real Exchange Rates: Empirical Evidence for OECD Countries," NBER working paper no. 6017 (1997).

Dufour, Jean-Marie, and O. Torres, "Union-Intersection and Sample-Split Methods in Econometrics with Application to MA and SURE Models," paper presented at the summer meetings of the Econometric Society, Iowa City (1996).
Frankel, Jeffrey, and Andrew Rose, "A Panel Project on Purchasing Power Parity: Mean Reversion Within and Between Countries," Journal of International Economics 40:1/2 (1996), 209-224.

Kao, Chihwa, and Min-Hsien Chiang, "On the Estimation and Inference of a Cointegrated Regression In Panel Data," Syracuse University manuscript (1997).

Maddala, G. S., and Shaowen Wu, "A Comparative Study of Unit Root Tests with Panel Data and a New Simple Test," Oxford Bulletin of Economics and Statistics 61:4 (1999), 631-652.

Mark, Nelson, and Donggyu Sul, "A Computationally Simple Cointegration Vector Estimator for Panel Data," Ohio State University manuscript (1999).

Obstfeld, Maurice, and Alan Taylor, "International Capital-Market Integration over the Long Run: The Great Depression as a Watershed," U.C. Berkeley manuscript (1996).

O'Connell, Paul, "The Overvaluation of Purchasing Power Parity," Journal of International Economics 44:1 (1998), 1-19.

Oh, Keun-Yeob, "Purchasing Power Parity and Unit Root Tests Using Panel Data," Journal of International Money and Finance 15:31 (1996), 405-418.

Papell, David, "Searching for Stationarily: Purchasing Power Parity Under the Current Float," Journal of International Economics 43:3/4 (1997), 313-332.

Pedroni, Peter, "Panel Cointegration: Asymptotic and Finite Sample Properties of Pooled Time Series Tests, With an Application to the PPP Hypothesis," Indian a University working papers in economics no. 95-013 (June 1995).

__ , "Fully Modified OLS for Heterogeneou s Cointegrated Panels and the Case of Purchasing Power Parity," Indiana University working papers in economics no. 96-020 (June 1996).

_ "On the Role of Cross Sectional Dependency in Panel Unit Root and Panel Cointegration Exchange Rate Studies," Indiana University manuscript (1997).

,"Fully Modified OLS for Heterogeneou s Cointegrated Panels," Advances in Econometrics 15 (2000), 93-130.

Phillips, Peter, and Hyungsik Moon, "Linear Regression Limit Theory for Nonstationar y Panel Data," Econometrica 67:5 (1999), 1057-1111.

Taylor, Alan, "International Capital Mobility in History: Purchasing Power Parity in the Long-Run," NBER working paper no. 5742 (1996).

Wu, Yangru, "Are Real Exchange Rates Nonstationary? Evidence from a Panel-Data Test," Journal of Money Credit and Banking 28:1 (1996), 54-63. 